\title{
Homo- and Heteroleptic Complexes of Four-membered Group 13 Metal(I) N- Heterocyclic Carbene Analogues with Group 10 Metal(0) Fragments
}

\author{
Shaun P. Green, Cameron Jones* and Andreas Stasch \\ SUPPORTING INFORMATION
}

Synthesis of compounds $3-6$.

Synthesis of $\left[\mathbf{N i}(\mathbf{C O D})\left\{\mathbf{G a}[\mathbf{N}(\mathbf{A r})]_{2} \mathbf{C N C y}_{2}\right\}_{2}\right]$ 3: A solution of $\mathbf{1}(0.20 \mathrm{~g}, 0.327 \mathrm{mmol})$ in toluene $(15 \mathrm{~mL})$ was added to a solution of $\left[\mathrm{Ni}(\mathrm{COD})_{2}\right](44 \mathrm{mg}, 0.159 \mathrm{mmol})$ in toluene $(3 \mathrm{~mL})$ at $-80^{\circ} \mathrm{C}$. The resultant solution was warmed to ambient temperature and stirred for $1 \mathrm{~h}$. It was then concentrated to $c a .5 \mathrm{~mL}$ and filtered. Slow cooling of the filtrate to $-30^{\circ} \mathrm{C}$ afforded large red blocks of 3. Yield: $0.14 \mathrm{~g}$ (63\%); Mp: $188-193{ }^{\circ} \mathrm{C}$ (dec.). ${ }^{1} \mathrm{H}$ NMR $\left(400 \mathrm{MHz}, 296 \mathrm{~K}, \mathrm{C}_{6} \mathrm{D}_{6}\right): \delta=0.60-2.24\left(\mathrm{~m}, 48 \mathrm{H}, \mathrm{COD}\right.$ and $\left.\mathrm{Cy}-\mathrm{CH}_{2}\right), 1.22\left(\mathrm{~d},{ }^{3} J_{\mathrm{HH}}=6.8 \mathrm{~Hz}\right.$, $\left.24 \mathrm{H}, \mathrm{CH}\left(\mathrm{CH}_{3}\right)_{2}\right), 1.38\left(\mathrm{~d},{ }^{3} J_{\mathrm{HH}}=6.8 \mathrm{~Hz}, 24 \mathrm{H}, \mathrm{CH}\left(\mathrm{CH}_{3}\right)_{2}\right), 3.51$ (m, $\left.4 \mathrm{H}, \mathrm{NCH}\right), 3.65$ (sept., $\left.{ }^{3} J_{\mathrm{HH}}=6.8 \mathrm{~Hz}, 8 \mathrm{H}, \mathrm{CH}\left(\mathrm{CH}_{3}\right)_{2}\right), 4.28($ br., $4 \mathrm{H}, \mathrm{COD} \mathrm{CH}), 6.88-7.22(\mathrm{~m}, 12 \mathrm{H}, \mathrm{ArH}) ;{ }^{13} \mathrm{C}\left\{{ }^{1} \mathrm{H}\right\}$ NMR (75.6 MHz, $\left.296 \mathrm{~K}, \mathrm{C}_{6} \mathrm{D}_{6}\right): \delta=22.1\left(\mathrm{Cy}-\mathrm{CH}_{2}\right), 25.6\left(\mathrm{Cy}-\mathrm{CH}_{2}\right), 26.8\left(\mathrm{CH}\left(\mathrm{CH}_{3}\right)_{2}\right), 27.1$ $\left(\mathrm{CH}\left(\mathrm{CH}_{3}\right)_{2}\right), 28.3\left(\mathrm{CH}\left(\mathrm{CH}_{3}\right)_{2}\right), 31.5\left(\mathrm{COD}-\mathrm{CH}_{2}\right), 35.2\left(\mathrm{Cy}-\mathrm{CH}_{2}\right), 58.7(\mathrm{NCH}), 71.7(\mathrm{COD}-$ $C H), 123.3(\mathrm{ArC}), 124.0(\mathrm{ArC}), 142.7(\mathrm{ArC}), 143.1(\mathrm{ArC}), 167.0\left(\mathrm{~N}_{3} C\right) ; \mathrm{IR} \mathrm{v} / \mathrm{cm}^{-1}$ (Nujol): 1613 (s), 1584 (m), 1328 (s), 1243 (m), 1162 (m), 1107 (m), 1017 (s); MS/EI m/z(\%): 544 [(ArN) $\left.)_{2} \mathrm{CNCy}_{2} \mathrm{H}^{+}, 100\right]$; anal. calc. for $\mathrm{C}_{82} \mathrm{H}_{124} \mathrm{Ga}_{2} \mathrm{~N}_{6} \mathrm{Ni}$ : C 70.75, H 8.98, N 6.04\%; found: C 70.13, H 8.75, N 5.98\%;

Crystals of 3.(THF) $)_{3}$ suitable for X-ray single crystal diffraction were obtained by recrystallisation from THF.

Synthesis of $\left[\mathbf{P t}(\mathbf{d p p e})\left\{\mathrm{Ga}[\mathbf{N}(\mathrm{Ar})]_{2} \mathrm{CNCy}_{2}\right\}_{2}\right]$ 4: A solution of $\mathbf{1}(0.25 \mathrm{~g}, 0.42 \mathrm{mmol})$ in toluene $(12 \mathrm{~mL})$ was added to a slurry of $\left[(\mathrm{dppe}) \mathrm{Pt}\left(\mathrm{C}_{2} \mathrm{H}_{4}\right)\right](0.11 \mathrm{~g}, 0.20 \mathrm{mmol})$ in toluene 
$(8 \mathrm{~mL})$ at $-80^{\circ} \mathrm{C}$. An immediate colour change to yellow-brown occurred. The reaction mixture was warmed to ambient temperature. Volatiles were then removed in vacuo and the brown residue extracted with hexane $(30 \mathrm{~mL})$. Concentration of the extract to $c a .12 \mathrm{~mL}$ and cooling to $-30^{\circ} \mathrm{C}$ afforded yellow blocks of 4 . Yield: $70 \mathrm{mg}$ (19\%); Mp: 90-93 deg (dec.).

${ }^{31} \mathrm{P}\left\{{ }^{1} \mathrm{H}\right\}$ NMR (on reaction solution): $\delta 39.9\left({ }^{1} J_{\mathrm{PPt}}=3860 \mathrm{~Hz}\right) ; \mathrm{IR} v / \mathrm{cm}^{-1}$ (Nujol):1614 (m), $1584(\mathrm{~m}), 1323$ (m), 1252 (m), 1152 (m), 1097 (m), 976 (m), 806 (m), 745 (m); MS/EI $\mathrm{m} / \mathrm{z}(\%): 544\left((\mathrm{ArN})_{2} \mathrm{CNCy}_{2} \mathrm{H}^{+}, 100\right)$.

Synthesis of $\left[\mathbf{P t}(\mathbf{d p p e})\left\{\operatorname{In}[\mathbf{N}(\mathbf{A r})]_{2} \mathbf{C N C y}_{2}\right\}_{2}\right]$ 5: A solution of $\mathbf{2}(0.25 \mathrm{~g}, 0.38 \mathrm{mmol})$ in toluene $(8 \mathrm{~mL})$ was added to a slurry of $\left[(\mathrm{dppe}) \mathrm{Pt}\left(\mathrm{C}_{2} \mathrm{H}_{4}\right)\right](0.10 \mathrm{~g}, 0.18 \mathrm{mmol})$ in toluene $(5 \mathrm{~mL})$ at $-80^{\circ} \mathrm{C}$. An immediate colour change to yellow-brown occurred. The reaction mixture was warmed to ambient temperature and volatiles were removed in vacuo. The brown residue was extracted with hexane $(20 \mathrm{~mL})$. Concentration of the extract to $c a .6 \mathrm{~mL}$ and cooling to $-30^{\circ} \mathrm{C}$ led to yellow blocks of 5. Yield: $60 \mathrm{mg}$ (17\%); Mp: 150-152 deg (dec.). IR v/cm ${ }^{-1}$ (Nujol): 1613 (m), 1585 (m), 1322 (m), 1253 (m), 1150 (m), 1097 (m), 976 (m), 746 (m); MS/EI m/z(\%): $544\left((\mathrm{ArN})_{2} \mathrm{CNCy}_{2} \mathrm{H}^{+}, 100\right)$.

NB: No solution state spectroscopic data could be obtained on pure samples of $\mathbf{4}$ and $\mathbf{5}$ because of their low stability upon dissolution in normal deuterated solvents. Similarly, reproducible analytical data could not be obtained on these compounds due to the presence of solvents of crystallization in both cases. In addition, crystalline samples of both were contaminated with small amounts of $\left[\mathrm{Pt}(\mathrm{dppe})_{2}\right]$ (a reaction by-product) which could not be completely separated from the samples manually.

Synthesis of $\left[\mathbf{P t}\left\{\mathbf{G a}[\mathbf{N}(\mathrm{Ar})]_{2} \mathbf{C N C y}_{2}\right\}_{3}\right]$ 6: A solution of $\mathbf{1}(0.15 \mathrm{~g}, 0.245 \mathrm{mmol})$ in toluene $(12 \mathrm{~mL})$ was added to a solution of $\left[\mathrm{Pt}(\text { norbornene })_{3}\right](39 \mathrm{mg}, 0.082 \mathrm{mmol})$ in toluene $(6 \mathrm{~mL})$ 
at $-80^{\circ} \mathrm{C}$. The solution was then warmed to ambient temperature and concentrated under reduced pressure to $c a .8 \mathrm{~mL}$. It was then filtered and the filtrate slowly cooled to $-30^{\circ} \mathrm{C}$ to afford orange blocks of 6.Yield 48\%; Mp: 161-163 deg (dec.). ${ }^{1} \mathrm{H}$ NMR (400 MHz, $296 \mathrm{~K}$, $\left.\mathrm{C}_{6} \mathrm{D}_{6}\right): \delta=0.52-1.68\left(\mathrm{~m}, 60 \mathrm{H}, \mathrm{CH}_{2}\right), 1.24\left(2 x b r\right.$. overlapping d, $72 \mathrm{H},{ }^{3} J_{\mathrm{HH}}=c a .7 \mathrm{~Hz}$, $\left.\mathrm{CH}\left(\mathrm{CH}_{3}\right)_{2}\right), 3.31$ (br. m, $\left.6 \mathrm{H}, \mathrm{NCH}\right), 3.47$ (br. sept., ${ }^{3} \mathrm{~J}_{\mathrm{HH}}=$ ca. $\left.7 \mathrm{~Hz}, 12 \mathrm{H}, \mathrm{CH}\left(\mathrm{CH}_{3}\right)_{2}\right), 6.78-$ $7.18(\mathrm{~m}, 18 \mathrm{H}, \mathrm{Ar} H) ;{ }^{13} \mathrm{C}\left\{{ }^{1} \mathrm{H}\right\} \operatorname{NMR}\left(75.6 \mathrm{MHz}, 296 \mathrm{~K}, \mathrm{C}_{6} \mathrm{D}_{6}\right): \delta=21.8,25.4$ (br., $\mathrm{CH}_{2}$ ), 27.9, 28.2 (br., $\left.\mathrm{CH}\left(\mathrm{CH}_{3}\right)_{2}\right)$, 28.5 (br., $\left.\mathrm{CH}\left(\mathrm{CH}_{3}\right)_{2}\right), 34.8\left(\mathrm{CH}_{2}\right), 58.7(\mathrm{CHN}), 123.1,124.4$, 143.8, 145.3 (ArC), 166.2 (br., $\mathrm{N}_{3} C$ ); IR v/cm ${ }^{-1}$ (Nujol): 1611 (s), 1583 (m), 1259 (m), 1160 (m), 1097 (m), 1019 (m), 953 (m), 895 (m); MS/EI m/z(\%): 544 [(ArN) $\left.{ }_{2} \mathrm{CNCy}_{2} \mathrm{H}^{+}, 100\right]$; anal. calc. for $\mathrm{C}_{111} \mathrm{H}_{168} \mathrm{Ga}_{3} \mathrm{~N}_{9} \mathrm{Pt}$ : C 65.58, H 8.33, N 6.20\%; found: C 64.98, H 8.15, N 6.08\%. 
Molecular structures of compounds 4 and 5.

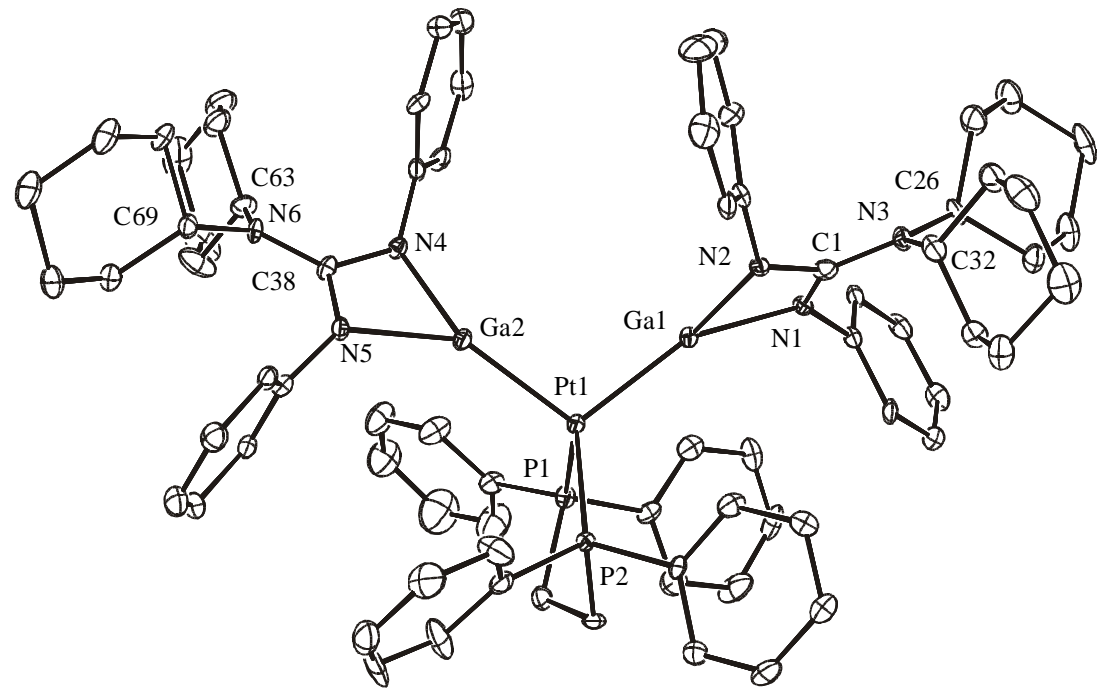

Fig. S1 Molecular structure of $\mathbf{4}$ (hydrogens and isopropyl groups omitted). Selected bond lengths $(\AA)$ and angles (o): $\mathrm{Pt}(1)-\mathrm{P}(2)$ 2.260(2), $\mathrm{Pt}(1)-\mathrm{P}(1)$ 2.262(2), $\mathrm{Pt}(1)-\mathrm{Ga}(2)$ 2.3549(11), Pt(1)-Ga(1) 2.3596(13), Ga(1)-N(2) 2.040(7), Ga(1)-N(1) 2.104(6), Ga(2)-N(4) 2.056(6), $\mathrm{Ga}(2)-\mathrm{N}(5)$ 2.090(6), N(1)-C(1) 1.338(10), N(2)-C(1) 1.351(9), N(3)-C(1) 1.386(10), N(4)$\mathrm{C}(38)$ 1.352(9), N(5)-C(38) 1.353(9), N(6)-C(38) 1.375(9), P(2)-Pt(1)-P(1) 89.32(8), P(2)Pt(1)-Ga(2) 117.99(6), P(1)-Pt(1)-Ga(2) 112.81(6), P(2)-Pt(1)-Ga(1) 113.57(6), P(1)-Pt(1)$\mathrm{Ga}(1)$ 118.93(7), Ga(2)-Pt(1)-Ga(1) 104.55(4), N(2)-Ga(1)-N(1) 64.2(2), N(4)-Ga(2)-N(5) 64.4(2), N(1)-C(1)-N(2) 109.8(7), N(4)-C(38)-N(5) 109.5(6). 


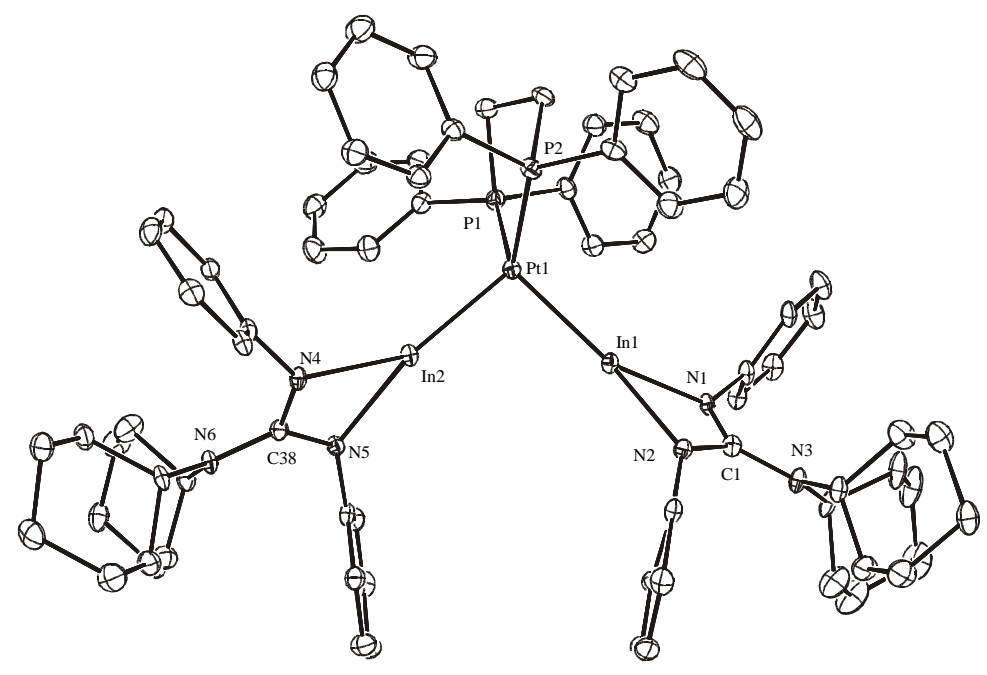

Fig. S2 Molecular structure of $\mathbf{5}$ (hydrogens and isopropyl groups omitted). Selected bond lengths ( $\AA$ ) and angles (O): $\operatorname{Pt}(1)-\mathrm{P}(1)$ 2.2489(18), $\operatorname{Pt}(1)-\mathrm{P}(2)$ 2.2500(19), $\operatorname{Pt}(1)-\operatorname{In}(1)$ 2.5329(10), $\operatorname{Pt}(1)-\operatorname{In}(2) \quad 2.5341(11), \operatorname{In}(1)-\mathrm{N}(2) \quad 2.231(5), \quad \operatorname{In}(1)-\mathrm{N}(1) \quad 2.306(5), \quad \operatorname{In}(2)-\mathrm{N}(5)$ 2.237(5), $\mathrm{In}(2)-\mathrm{N}(4)$ 2.302(5), N(1)-C(1) 1.357(8), N(2)-C(1) 1.362(8), N(3)-C(1) 1.372(9), $\mathrm{N}(4)-\mathrm{C}(38)$ 1.344(8), N(5)-C(38) 1.347(8), N(6)-C(38) 1.388(8), P(1)-Pt(1)-P(2) 90.60(7), $\mathrm{P}(1)-\mathrm{Pt}(1)-\operatorname{In}(1)$ 115.24(6), $\mathrm{P}(2)-\mathrm{Pt}(1)-\operatorname{In}(1)$ 119.83(6), $\mathrm{P}(1)-\mathrm{Pt}(1)-\operatorname{In}(2)$ 119.19(6), $\mathrm{P}(2)-\mathrm{Pt}(1)-$ $\operatorname{In}(2) \quad 116.22(6), \quad \operatorname{In}(1)-\operatorname{Pt}(1)-\operatorname{In}(2) \quad 97.59(3), \quad \mathrm{N}(2)-\operatorname{In}(1)-\mathrm{N}(1) \quad 59.33(19), \quad \mathrm{N}(5)-\operatorname{In}(2)-\mathrm{N}(4)$ 58.9(2), N(1)-C(1)-N(2) 111.4(6), N(4)-C(38)-N(5) 112.1(6). 


\section{Theoretical study}

Method: The geometry of the model complex, $\left[\mathrm{Pt}\left\{\mathrm{Ga}\left[\mathrm{N}\left(\mathrm{C}_{6} \mathrm{H}_{3} \mathrm{Me}_{2}-2,6\right)\right]_{2} \mathrm{CNMe}_{2}\right\}_{3}\right]$, was optimised using the Gaussian 98 package ${ }^{1}$ employing the methods recommended by Boehme and Frenking. ${ }^{2}$ That is, the BP86 density functional method, ${ }^{3}$ with a $6-31 \mathrm{G}^{*}$ basis set on $\mathrm{C}, \mathrm{N}$ and $\mathrm{H},{ }^{4}$ Stuttgart-Dresden ECP/basis sets for $\mathrm{Ga}$ and $\mathrm{Pt},{ }^{5}$ augmented by a d-type polarization function with exponent 0.207 on $\mathrm{Ga}^{6}{ }^{6}$ Dative and $\pi$-bonding components of the Pt-Ga bonds were obtained by charge decomposition analysis, using CDA version 2.1.2. ${ }^{7}$

1. Frisch, M. J.; Trucks, G. W.; Schlegel, H. B.; Scuseria, G. E.; Robb, M. A.; Cheeseman, J. R.; Montgomery, Jr., J. A.; Vreven, T.; Kudin, K. N.; Burant, J. C.; Millam, J. M.; Iyengar, S. S.; Tomasi, J.; Barone, V.; Mennucci, B.; Cossi, M.; Scalmani, G.; Rega, N.; Petersson, G. A.; Nakatsuji, H.; Hada, M.; Ehara, M.; Toyota, K.; Fukuda, R.; Hasegawa, J.; Ishida, M.; Nakajima, T.; Honda, Y.; Kitao, O.; Nakai, H.; Klene, M.; Li, X.; Knox, J. E.; Hratchian, H. P.; Cross, J. B.; Adamo, C.; Jaramillo, J.; Gomperts, R.; Stratmann, R. E.; Yazyev, O.; Austin, A. J.; Cammi, R.; Pomelli, C.; Ochterski, J. W.; Ayala, P. Y.; Morokuma, K.; Voth, G. A.; Salvador, P.; Dannenberg, J. J.; Zakrzewski, V. G.; Dapprich, S.; Daniels, A. D.; Strain, M. C.; Farkas, O.; Malick, D. K.; Rabuck, A. D.; Raghavachari, K.; Foresman, J. B.; Ortiz, J. V.; Cui, Q.; Baboul, A. G.;. Clifford, S.; Cioslowski, J.; Stefanov, B. B.; Liu, G.; Liashenko, A.; Piskorz, P.; Komaromi, I.; Martin, R. L.; Fox, D. J.; Keith, T.; AlLaham, M. A.; Peng, C. Y.; Nanayakkara, A.; Challacombe, M.; Gill, P. M. W.;

Johnson, B.; Chen, W.; Wong, M. W.; Gonzalez, C.; Pople, J. A. Gaussian 98, Revision A.10, Gaussian, Inc., Pittsburgh PA, 2001.

2. Boehme, C.; Frenking, G. Chem. Eur. J. 1999, 5, 7.

3. (a) Becke, A.D. Phys. Rev. A 1988, 38, 3098; (b) Perdew, J.P. Phys. Rev. A 1986, 33, 8822.

4. (a) Ditchfield, R.; Hehre, W.J.; Pople, J.A. J. Chem. Phys. 1971, 54, 724; (b) Hehre, W.J.; Ditchfield, R.; Pople, J.A. J. Chem. Phys. 1972, 56, 2257; (c) Gordon, M.S. Chem. Phys. Lett. 1980, 76, 163; (d) Hariharan, P.C.; Pople, J.A. Theor. Chim. Acta 1973, 28, 213.

5. (a) Dolg, M.; Wedig, U.; Stoll, H.; Preuss, H. J. Chem. Phys. 1987, 86, 866; (b) Bergner, A.; Dolg, M.; Kuehle, W.; Stoll, H.; Preuss, H. Mol. Phys. 1993, 80, 1431. 
6. Andzelm, J.; Huzinaga, S.; Klobukowski, M.; Radzio, E.; Sakai, Y.; Tatekawi, H. Gaussian Basis Sets for Molecular Calculations, Elsevier, Amsterdam, 1984.

7. Dapprich S.; Frenking G. J. Phys. Chem., 1995, 99, 9352.

\section{Cartesian coordinates of the optimised structure of $\left[\mathrm{Pt}\left\{\mathrm{Ga}\left[\mathrm{N}\left(\mathrm{C}_{6} \mathrm{H}_{3} \mathrm{Me}_{2}-2,6\right)\right]_{2} \mathrm{CNMe}\right\}_{3}\right]$.}

\begin{tabular}{|c|c|c|c|}
\hline $\mathrm{C}$ & -3.81531 & 4.50620 & -3.07250 \\
\hline $\mathrm{C}$ & -2.50655 & 4.24214 & -2.63379 \\
\hline C & -2.14606 & 4.56471 & -1.29340 \\
\hline C & -3.12280 & 5.08751 & -0.39638 \\
\hline $\mathrm{C}$ & -4.41950 & 5.34307 & -0.88360 \\
\hline C & -4.76998 & 5.06687 & -2.21160 \\
\hline $\mathrm{C}$ & -2.81115 & 5.33052 & 1.06548 \\
\hline $\mathrm{N}$ & -0.82910 & 4.23980 & -0.88338 \\
\hline $\mathrm{C}$ & 0.01282 & 5.01688 & -0.15065 \\
\hline $\mathrm{N}$ & 0.00473 & 6.39192 & -0.17383 \\
\hline C & -0.59722 & 7.15268 & -1.26840 \\
\hline $\mathrm{C}$ & -1.49011 & 3.62050 & -3.56627 \\
\hline $\mathrm{N}$ & 0.86338 & 4.27441 & 0.60662 \\
\hline $\mathrm{Ga}$ & 0.01877 & 2.43638 & -0.09671 \\
\hline Pt & 0.01659 & 0.04855 & -0.02973 \\
\hline $\mathrm{Ga}$ & -1.98011 & -1.13646 & 0.52557 \\
\hline $\mathrm{N}$ & -3.72979 & -2.08393 & -0.25549 \\
\hline $\mathrm{C}$ & -4.51042 & -1.85522 & -1.41632 \\
\hline $\mathrm{C}$ & -4.07350 & -2.47519 & -2.62259 \\
\hline $\mathrm{C}$ & -4.78990 & -2.24175 & -3.80900 \\
\hline $\mathrm{C}$ & -5.91249 & -1.40123 & -3.82463 \\
\hline $\mathrm{C}$ & -6.31150 & -0.76456 & -2.64238 \\
\hline $\mathrm{C}$ & -5.62073 & -0.96233 & -1.43074 \\
\hline $\mathrm{C}$ & -2.85352 & -3.37003 & -2.61947 \\
\hline $\mathrm{C}$ & -6.03686 & -0.19248 & -0.19530 \\
\hline $\mathrm{N}$ & -3.31787 & -2.00456 & 1.95951 \\
\hline $\mathrm{C}$ & -4.17434 & -2.41383 & 0.98451 \\
\hline $\mathrm{N}$ & -5.35669 & -3.07210 & 1.23086 \\
\hline $\mathrm{C}$ & -6.03138 & -3.88306 & 0.21749 \\
\hline $\mathrm{C}$ & -3.10993 & -2.61453 & 3.22106 \\
\hline $\mathrm{C}$ & -2.89824 & -4.01630 & 3.37375 \\
\hline $\mathrm{C}$ & -2.65670 & -4.52589 & 4.66472 \\
\hline $\mathrm{C}$ & -2.59583 & -3.68610 & 5.78430 \\
\hline $\mathrm{C}$ & -2.76284 & -2.30357 & 5.61715 \\
\hline $\mathrm{C}$ & -3.01274 & -1.74997 & 4.35002 \\
\hline $\mathrm{C}$ & -2.88786 & -4.95384 & 2.18481 \\
\hline $\mathrm{C}$ & -3.17897 & -0.25625 & 4.17805 \\
\hline $\mathrm{C}$ & -6.01724 & -3.02912 & 2.53524 \\
\hline $\mathrm{Ga}$ & 2.01022 & -1.18417 & -0.48965 \\
\hline $\mathrm{N}$ & 3.25905 & -2.32964 & -1.80677 \\
\hline $\mathrm{C}$ & 2.96585 & -3.13896 & -2.93232 \\
\hline $\mathrm{C}$ & 2.65930 & -4.52693 & -2.82399 \\
\hline $\mathrm{C}$ & 2.33637 & -5.24077 & -3.99475 \\
\hline $\mathrm{C}$ & 2.28436 & -4.61150 & -5.24549 \\
\hline $\mathrm{C}$ & 2.54231 & -3.23574 & -5.33421 \\
\hline $\mathrm{C}$ & 2.87645 & -2.48438 & -4.19455 \\
\hline $\mathrm{C}$ & 2.63130 & -5.23172 & -1.48430 \\
\hline $\mathrm{C}$ & 3.14164 & -0.99841 & -4.29854 \\
\hline $\mathrm{C}$ & 4.14283 & -2.60275 & -0.80909 \\
\hline $\mathrm{N}$ & 3.77761 & -2.02711 & 0.36606 \\
\hline $\mathrm{C}$ & 4.62901 & -1.61624 & 1.42182 \\
\hline C & 4.22894 & -1.95238 & 2.74753 \\
\hline
\end{tabular}




\begin{tabular}{|c|c|c|c|}
\hline C & 5.02204 & -1.52975 & 3.82821 \\
\hline C & 6.18590 & -0.77473 & 3.62240 \\
\hline C & 6.55018 & -0.41479 & 2.31864 \\
\hline C & 5.78320 & -0.80878 & 1.20474 \\
\hline $\mathrm{C}$ & 2.96611 & -2.75191 & 2.98330 \\
\hline C & 6.17239 & -0.33274 & -0.17883 \\
\hline $\mathrm{N}$ & 5.27251 & -3.37057 & -0.97235 \\
\hline C & 5.86721 & -3.62009 & -2.28512 \\
\hline $\mathrm{C}$ & 5.95892 & -4.00389 & 0.15391 \\
\hline $\mathrm{C}$ & 2.17509 & 4.62676 & 1.01085 \\
\hline C & 2.53536 & 4.34657 & 2.36081 \\
\hline C & 3.83930 & 4.63887 & 2.79542 \\
\hline C & 4.78976 & 5.18651 & 1.92144 \\
\hline $\mathrm{C}$ & 4.43984 & 5.42171 & 0.58568 \\
\hline C & 3.14773 & 5.13712 & 0.10225 \\
\hline C & 1.52344 & 3.73932 & 3.30758 \\
\hline $\mathrm{C}$ & 2.83754 & 5.33663 & -1.36622 \\
\hline $\mathrm{C}$ & 0.59023 & 7.19607 & 0.89845 \\
\hline $\mathrm{H}$ & -1.90977 & 3.49851 & -4.57968 \\
\hline $\mathrm{H}$ & -0.57122 & 4.23135 & -3.64091 \\
\hline $\mathrm{H}$ & -1.17095 & 2.62273 & -3.20941 \\
\hline $\mathrm{H}$ & -3.74256 & 5.39552 & 1.65371 \\
\hline $\mathrm{H}$ & -2.19815 & 4.51335 & 1.48714 \\
\hline $\mathrm{H}$ & -2.24810 & 6.26859 & 1.23307 \\
\hline $\mathrm{H}$ & -4.08514 & 4.26364 & -4.10770 \\
\hline $\mathrm{H}$ & -5.78629 & 5.26883 & -2.56804 \\
\hline $\mathrm{H}$ & -5.17018 & 5.74846 & -0.19322 \\
\hline $\mathrm{H}$ & -2.94834 & -4.19255 & -1.88603 \\
\hline $\mathrm{H}$ & -2.68704 & -3.81306 & -3.61646 \\
\hline $\mathrm{H}$ & -1.94128 & -2.80975 & -2.34014 \\
\hline $\mathrm{H}$ & -6.73118 & 0.62204 & -0.46297 \\
\hline $\mathrm{H}$ & -6.54236 & -0.82801 & 0.55652 \\
\hline $\mathrm{H}$ & -5.16306 & 0.26050 & 0.30813 \\
\hline $\mathrm{H}$ & -7.16684 & -0.07723 & -2.65336 \\
\hline $\mathrm{H}$ & -6.46112 & -1.22876 & -4.75741 \\
\hline $\mathrm{H}$ & -4.45616 & -2.72897 & -4.73348 \\
\hline $\mathrm{H}$ & -2.37870 & -5.89883 & 2.44119 \\
\hline $\mathrm{H}$ & -3.90672 & -5.21182 & 1.83773 \\
\hline $\mathrm{H}$ & -2.36281 & -4.50672 & 1.32163 \\
\hline $\mathrm{H}$ & 2.83962 & -2.98012 & 4.05562 \\
\hline $\mathrm{H}$ & 2.06810 & -2.20041 & 2.64563 \\
\hline $\mathrm{H}$ & 2.97096 & -3.70608 & 2.42392 \\
\hline $\mathrm{H}$ & 6.87936 & 0.51183 & -0.11227 \\
\hline $\mathrm{H}$ & 6.65626 & -1.12391 & -0.78307 \\
\hline $\mathrm{H}$ & 5.28949 & 0.00737 & -0.74995 \\
\hline $\mathrm{H}$ & 2.10577 & -4.62758 & -0.72241 \\
\hline $\mathrm{H}$ & 2.11165 & -6.20174 & -1.56739 \\
\hline $\mathrm{H}$ & 3.64445 & -5.43137 & -1.08669 \\
\hline $\mathrm{H}$ & 3.05429 & -0.65508 & -5.34381 \\
\hline $\mathrm{H}$ & 2.42739 & -0.41662 & -3.68595 \\
\hline $\mathrm{H}$ & 4.15023 & -0.73302 & -3.92973 \\
\hline $\mathrm{H}$ & -3.14955 & 0.25864 & 5.15395 \\
\hline $\mathrm{H}$ & -2.37922 & 0.17068 & 3.54357 \\
\hline $\mathrm{H}$ & -4.13355 & -0.00375 & 3.67978 \\
\hline $\mathrm{H}$ & -2.69919 & -1.63472 & 6.48438 \\
\hline $\mathrm{H}$ & -2.40096 & -4.10404 & 6.77831 \\
\hline $\mathrm{H}$ & -2.49400 & -5.60485 & 4.78198 \\
\hline $\mathrm{H}$ & 7.43891 & 0.20679 & 2.15145 \\
\hline $\mathrm{H}$ & 6.79400 & -0.45214 & 4.47502 \\
\hline $\mathrm{H}$ & 4.71612 & -1.79999 & 4.84646 \\
\hline $\mathrm{H}$ & 2.48491 & -2.72815 & -6.30502 \\
\hline
\end{tabular}




$\begin{array}{rrrr}\mathrm{H} & 2.02638 & -5.18545 & -6.14254 \\ \mathrm{H} & 2.10220 & -6.30958 & -3.91063 \\ \mathrm{H} & 1.94353 & 3.64515 & 4.32374 \\ \mathrm{H} & 0.59958 & 4.34461 & 3.36774 \\ \mathrm{H} & 1.21295 & 2.73086 & 2.97420 \\ \mathrm{H} & 3.76977 & 5.40858 & -1.95233 \\ \mathrm{H} & 2.24491 & 4.49582 & -1.77019 \\ \mathrm{H} & 2.25379 & 6.25695 & -1.55819 \\ \mathrm{H} & 5.18748 & 5.81692 & -0.11380 \\ \mathrm{H} & 5.80258 & 5.40945 & 2.27524 \\ \mathrm{H} & 4.10936 & 4.42846 & 3.83753 \\ \mathrm{H} & 0.08321 & 7.98072 & -1.54276 \\ \mathrm{H} & -0.73932 & 6.50781 & -2.14619 \\ \mathrm{H} & -1.57839 & 7.58109 & -0.98545 \\ \mathrm{H} & -0.11029 & 8.01310 & 1.15545 \\ \mathrm{H} & 0.75348 & 6.57810 & 1.79167 \\ \mathrm{H} & 1.55821 & 7.64262 & 0.59885 \\ \mathrm{H} & 6.20603 & -5.04669 & -0.12109 \\ \mathrm{H} & 6.89619 & -3.47622 & 0.41726 \\ \mathrm{H} & 5.30764 & -4.01751 & 1.03813 \\ \mathrm{H} & 6.96574 & -3.51791 & -2.20305 \\ \mathrm{H} & 5.63340 & -4.63568 & -2.65961 \\ \mathrm{H} & 5.49935 & -2.88635 & -3.01510 \\ \mathrm{H} & -6.34390 & -4.84029 & 0.67646 \\ \mathrm{H} & -6.92867 & -3.37574 & -0.18693 \\ \mathrm{H} & -5.34837 & -4.09803 & -0.61513 \\ \mathrm{H} & -7.10297 & -2.88545 & 2.37828 \\ \mathrm{H} & -5.86469 & -3.96329 & 3.10995 \\ \mathrm{H} & -5.63314 & -2.18774 & 3.12806\end{array}$

